\title{
Continuous versus Single-Injection Femoral Nerve Block for Total Knee Arthroplasty- A Meta-Analysis of Randomized Control Trials
}

\author{
Andrew B. Maye, Eoghan T. Hurley, Utkarsh Anil, Cathal J. Moran, Gerard Curley.
}

\section{Introduction:}

Total knee arthroplasty is among the most common procedures performed, and with ageing populations and increasing obesity the incidence is projected to increase massively in the future ${ }^{1}$. Postoperative knee pain can result in increased morbidity, length of hospital stay and decreased patient satisfaction ${ }^{2}$. Additionally, postoperative knee pain can delay rehabilitation and recovery. It has been recently shown that femoral nerve block (FNB) is superior to patient controlled analgesia with opioids and that it results in similar outcomes to continuous epidural analgesia with fewer side effects ${ }^{3}$.

Methods:

Two independent reviewers performed the literature search according to the PRISMA guidelines and each reviewed the results. The following search terms were used in MEDLINE, EMBASE, and The Cochrane Library, databases: (continous femoral nerve block or cfnb) AND (single femoral nerve block or single injection femoral nerve block or sfnb) AND (total knee arthroplasty or tka or knee arthroplasty or total knee replacement or tkr or knee replacement). The inclusion criteria were the following: 1) randomized control trial, 2) comparing cFNB and sFNB, 3) TKA, 4) published in a peer-reviewed journal, and 5) published in English. The exclusion criteria were the following: 1) non-randomized, 2) retrospective, 3) non-arthroplasty, 4) hip arthoplasty, or 5) abstract only. The quality of the studies was assessed using the Cochrane Collaboration risk of bias tool. The outcomes measured focused on Visual Analogue Scale, opioid consumption and length of hospital stay. All statistical analysis was performed using Review Manager ((RevMan) [Macintosh]. Version 5.3. Copenhagen: The Nordic Cochrane Centre, The Cochrane Collaboration, 2014.). Results were presented as risk ratio (RR) for dichotomous outcomes and mean difference (MD) for continuous outcomes, with a $95 \%$ confidence interval $(95 \% \mathrm{CI})$. A pvalue of $<0.05$ was considered to be statistically significant.

Results:

Eight randomized control trials with 616 patients were included in this review.

Opioid Consumption:

In the first 24 hours there was a statistically significant difference in opioid consumption in favor of cFNB $(p=0.03)$.

1) Kurtz S, Ong K, Lau E, Mowat F, Halpern M. Projections of primary and revision hip and knee arthroplasty in the United States from 2005 to 2030. J Bone Joint Surg Am 2007;89:780-5

2) Feng JE, Novikov D, Anoushiravani AA, Schwarzkopf R. Total knee arthroplasty: improving outcomes with a multidisciplinary approach. J Multidiscip Healthc. 2018; 11: 63-73.

3) Chan EY, Fransen M, Parker DA, Assam PN, Chua N. Femoral nerve blocks for acute postoperative pain after knee replacement surgery. Cochrane Database Syst Rev. 2014 May 13;(5): CD00994I.

4) Namba RS, Inacio MCS, Pratt NL, Graves SE, Roughead EE, Paxton EW. Persistent opioid use following total knee arthroplasty: a signal for close surveillance. J Arthroplasty 2018;33:33le6.
Visual Analogue Scale:

In the first 2 hours and 3-12 hours, there was no statistically significant difference in the VAS scores ( $p=0.39$, and $p=0.86$ respectively). At 24 hours, there was a statistically significant difference in the VAS scores in favor of cFNB at rest and on movement $(p=0.02$, and $p=$ 0.00001 respectively). At 48 hours, there was a statistically significant difference in the VAS scores in favor of cFNB on movement $(, p=0.02)$ but not at rest $(\mathrm{p}=0.05)$.

Hospital Stay:

There was no statistically significant difference in the length of hospital stay $(\mathrm{p}=0.94)$.

\section{Discussion:}

The most important finding from our meta-analysis of 8 randomized control trials was that patients receiving cFNB reported lower pain levels and had decreased opioid consumption following TKA compared to sFNB. Adequate pain relief reduces morbidity and increases patient satisfaction ${ }^{2}$. Opioid consumption is a significant problem in modern healthcare, with increasing rates in opioid addiction in recent years.

\begin{tabular}{|l|l|l|l|}
\hline OUTCOME & & FAVORS & P value \\
\hline VAS at 2 hours & MD cFNB vs sFNB $(95 \% \mathrm{Cl})$ & Neither & 0.39 \\
\hline VAS at 3-12 hours & MD cFNB vs sFNB $(95 \% \mathrm{Cl})$ & Neither & 0.86 \\
\hline $\begin{array}{l}\text { VAS at } 24 \text { hours on } \\
\text { movement }\end{array}$ & MD cFNB vs sFNB $(95 \% \mathrm{Cl})$ & cFNB & $0.0000 \mathrm{I}$ \\
\hline VAS at 24 hours at rest & MD cFNB vs sFNB $(95 \% \mathrm{Cl})$ & cFNB & 0.02 \\
\hline $\begin{array}{l}\text { VAS at } 48 \text { hours on } \\
\text { movement }\end{array}$ & MD cFNB vs sFNB $(95 \% \mathrm{Cl})$ & cFNB & 0.02 \\
\hline VAS at 48 hours at rest & MD cFNB vs sFNB $(95 \% \mathrm{Cl})$ & Neither & 0.05 \\
\hline $\begin{array}{l}\text { Opioid usage in first } 24 \\
\text { hours }\end{array}$ & MD cFNB vs sFNB $(95 \% \mathrm{Cl})$ & cFNB & 0.03 \\
\hline Length of hospital stay & MD cFNB vs sFNB $(95 \% \mathrm{Cl})$ & Neither & 0.94 \\
\hline
\end{tabular}

As well as the social benefit of decreasing opioid usage chronic opioid use following TKA is an independent risk factor for revision ${ }^{4}$. Another benefit of cFNB is that due to the use of shorter acting agents and dose modulation it can reduce systemic toxicity and neurotoxicity compared to $\mathrm{SFNB}$.

There are several limitations to this study. There was some heterogeneity in the outcome measures, which made analysis difficult. Pain is a variable and subjective outcome and so is difficult to measure consistently.

Conclusion:

This study has found that cFNB resulted in decreased pain levels and opioid consumption compared to sFNB following total knee arthroplasty. There was no significant difference in length of hospital stay. 